\title{
LA PROTECCIÓN CIVIL EN LAS INSTITUCIONES DE EDUCACIÓN PRIMARIA VALLENSE UN DESAFÍO PENDIENTE DE RESOLVER
}

\author{
CIVIL PROTECTION IN ELEMENTARY EDUCATION INSTITUTIONS IN VALLES, \\ A PENDING CHALLENGE TO BE SOLVED
}

Recibido: 24 de septiembre 2021

Aceptado: 6 de noviembre 2021

\author{
M.C. Rosas Rivera ${ }^{1}$ \\ F. Orduña Correa ${ }^{2}$ \\ L.A. Gutierrez Ramos ${ }^{3}$ \\ M.Y. García Hernández ${ }^{4}$
}

\section{RESUMEN}

México es considerado uno de los países más poblados del mundo y también donde ocurre un mayor número de desastres, por las características de su geografía la Organización de las Naciones Unidas en 2017 lo clasifica dentro de los treinta países más expuestos a sufrir los embates de la naturaleza. Altos han sido los costos de pérdidas humanas y materiales y graves los daños a los ecosistemas; con los aumentos de las temperaturas en los años recientes se han recrudecido los impactos de los fenómenos geofísicos y climatológicos en todos los rincones del país. La zona huasteca dentro del estado de San Luis Potosí es altamente susceptible a presentar cada vez con mayor frecuencia y en menor escala de tiempo efectos devastadores por las inundaciones y los incendios forestales, sumado a nuevos eventos que eran considerados en el pasado prácticamente improbables como lo son los sismos y la violencia social y hoy en día con la pandemia del covid 19, la colocan en una situación altamente vulnerable. Estas circunstancias han alcanzado al ámbito escolar, en donde la integridad de los niños y las niñas, así como la de los adultos depende de los esfuerzos encaminados a lograr el fortalecimiento de una cultura preventiva en materia de Protección Civil. Ya se cuenta con los ordenamientos legales y reglamentarios para implementar programas de Protección Civil, solo se requiere el impulso de las autoridades municipales, estatales y federales para hacer de la protección civil una actividad común a la vida cotidiana.

\section{PALABRAS CLAVE: Protección Civil Desastres Naturales Educación Primaria}

\section{ABSTRACT}

Mexico is considered one of the most populated countries in the world and also where a greater number of disasters occur, due to the characteristics of its geography the United Nations Organization in 2017 classified it within the thirty countries most exposed to suffering the onslaught of nature. High have been the costs of human and material losses and serious damage to ecosystems; with the increases in temperatures in recent years, the impacts of geophysical and climatological phenomena have worsened in all corners of the country. The Huasteca area within the state of San Luis Potosí is highly susceptible to presenting with increasing frequency and on a smaller scale of time devastating effects due to floods and forest fires, added to new events that were considered practically improbable in the past. they are earthquakes and social violence and today with the covid 19 pandemic, they place it in a highly vulnerable situation. These circumstances have reached the school environment, where the integrity of boys and girls, as well as that of adults, depends on efforts aimed at strengthening a preventive culture in matters of Civil Protection. The legal and regulatory regulations are already in place to implement Civil Protection programs, only the impulse of municipal, state and federal authorities is required to make civil protection an activity common to daily life.

KEY WORDS: Civil Protection Natural Disasters Primary Education

\footnotetext{
${ }^{1}$ Profesor del Tecnológico Nacional de México, Campus Ciudad Valles, carmen.rosas@tecvalles.mx

${ }^{2}$ Profesor del Tecnológico Nacional de México, Campus Ciudad Valles, francisco.orduna @ tecvalles.mx

${ }^{3}$ Director del Centro Integral de Capacitación y Estudios de Seguridad e Higiene en el Trabajo cicesh_00125@ hotmail.com

${ }^{4}$ Estudiante de IGE 18690243@tecvalles.mx
} 


\section{INTRODUCCIÓN}

En los últimos años los fenómenos naturales en México han sido cada vez más frecuentes causando grandes estragos para la población, afectando el patrimonio de los ciudadanos, poniendo en riesgo la salud y vulnerando la seguridad de la supervivencia humana.

Hemos presenciado a lo largo y ancho del país eventos de diversa índole, desde sismos de diversa intensidad que en la actualidad ocurren en áreas consideradas como atípicas, cuyos fenómenos estaban lejos de contemplarse como eventos riesgosos para la población, es el caso del estado de San Luis Potosí, donde de acuerdo al Servicio Sismológico Nacional (SSN) se tiene antecedente de que cada año ocurren de uno a cuatro sismos de entre 4 y 5 grados de magnitud; en lo que va del año 2021 se tiene registro de veintidós sismos cuya magnitud osciló entre los 3.3 y 4.5 grados (SSN 2021). Particularmente en Ciudad Valles, S.L.P., de acuerdo con las estadísticas registradas por el SSN existe un antecedente de actividad sísmica desde 1976 con magnitud no calculable, es decir que ha pasado desapercibido para la población, sin embargo, en este último año 2021 los sismos registrados para esta localidad han alcanzado una magnitud de cuatro grados de acuerdo al SSN.

Por otra parte, existen otros eventos naturales que han sido recurrentes, y que son atribuibles a los cambios climáticos y al aumento global de las temperaturas como lo son los ciclones, los huracanes, las ventiscas, los tornados, entre otros; algunos de ellos causando inundaciones severas que han provocado perjuicios económicos con serias afectaciones al patrimonio familiar, en otros casos, daños a las personas ocasionando deterioro de la salud y pérdidas humanas. Con relación a los incendios forestales se estima que el $99 \%$ de éstos son causados por las actividades humanas y el resto tiene su causa en fenómenos naturales, como son las descargas eléctricas y la erupción de los volcanes (CONAFOR 2010). A estas ocurrencias se suma el clima de inseguridad pública y violencia que actualmente enfrenta la sociedad, difundiendo el temor y la incertidumbre y causando cuantiosas pérdidas económicas.

Estas circunstancias han instituido la cultura de prevención en los diferentes organismos de la sociedad civil, anticipándose a mitigar los impactos de los desastres naturales y antropogénicos mediante la participación responsable, el involucramiento de las entidades gubernamentales y el despliegue de normativas y reglamentos oficiales dirigidos a generar estándares de desempeño capaces de responder a las contingencias y emergencias que eventualmente se tienen que enfrentar. La protección Civil es un término que ha tomado relevancia en nuestro país a partir de los sismos ocurridos en 1985 donde nos sorprendió la catástrofe sin estar preparados para responder de manera eficaz a las emergencias, pese a que en el pasado, habían ocurrido sismos de igual o mayor magnitud, a partir de éste evento las acciones de protección civil se volvieron una exigencia tanto para el desarrollo de infraestructura en la construcción de inmuebles como para el desenvolvimiento de la población, en los quehaceres de su vida cotidiana.

La Protección civil tiene sus orígenes en agosto 12 de 1949 en el Protocolo 1 adicional al tratado de Ginebra para la protección de víctimas de guerra de los conflictos armados internacionales (CICR 1977), fue definida como el "cumplimiento de algunas o de todas las tareas humanitarias que están destinadas a proteger a la población civil contra los peligros de las hostilidades y de las catástrofes y a ayudarla a recuperarse de sus efectos inmediatos, así como facilitar las condiciones necesarias para su supervivencia” (CICR 1977). En México en 1986 se crea Sistema Nacional de Protección Civil (SINAPROC) y en 1988 el Centro 
Nacional de Prevención de Desastres CENAPRED, (CENAPRED 2016); surge en el año 2000 la primera ley General de Protección Civil, abrogada y emitida nuevamente en el año 2012, con el objetivo de "establecer las bases de coordinación entre los distintos órdenes de gobierno en materia de protección civil" (PCGM 2012). se promulga también el reglamento de la Ley General de Protección Civil en el 2014 que comprende las políticas públicas aplicables en los tres órdenes de gobierno en esta materia (PCGM 2015).

Entre los impactos catastróficos más dolorosos que han afectado a la población infantil en la historia reciente de México, se encuentra el caso del incendio en la guardería ABC en Hermosillo Sonora en el año 2009 donde 49 niños perdieron la vida y más de 80 resultaron gravemente heridos, (CNDH-2018). En el ámbito escolar en las instituciones de educación básica; la Secretaría de Educación Pública, la Secretaría de Gobernación y el Instituto de la Infraestructura Física Educativa en coordinación con el Sistema Nacional de protección civil desarrollaron la guía para elaborar y o actualizar el programa interno de protección civil escolar, que tiene como propósito salvaguardar la integridad física y emocional de los niños, niñas y adolescentes (NNA), y que cada centro escolar tenga conocimiento de las situaciones de riesgo que puede enfrentar y se encuentren preparados para resolverlas mitigando su impacto. Se pretende que estas actividades formen parte de los "aprendizajes claves" de acuerdo a la propuesta curricular del modelo educativo. (INIFED 2018).

Teniendo como marco normativo la Constitución Política de los Estados Unidos Mexicanos, y como antecedente la convención de los derechos del niño, instituida en 1989 (UNICEF 2006), surge la iniciativa de la NOM-009-SEGOB-2015 Medidas de previsión, prevención y mitigación de riesgos en los centros de atención infantil en la modalidad pública, privada y mixta, y con ella la gobernanza en materia de protección civil para todas las instituciones y organismos dedicados a la atención de la niñez mexicana. Con éstos lineamiento de referencia se realiza esta investigación.

\section{METODOLOGÍA}

Para llevar a cabo este proyecto de investigación aplicada se realiza un análisis cualitativo y cuantitativo de las necesidades existentes en materia de protección civil y seguridad e higiene en las instituciones de educación básica de Ciudad Valles, S.L.P., encontrando que el tema era de interés para los responsables de los centros educativos ya que no se tenía conocimiento ni se habían realizado acciones encaminadas a esos objetivos. En deliberaciones realizadas con la Unidad Regional de Servicios Educativos Huasteca Norte (URSE) sobre los beneficios que se obtendrían, se acordó implementar un Programa Interno de Protección Civil en una escuela primaria localizada en el municipio de Ciudad Valles, S.L.P. con reconocida trayectoria académica y que además mostró interés y disponibilidad por parte del director que se encuentra al frente del plantel. Se lleva a cabo un plan de trabajo considerando la disponibilidad de tiempo del personal de la institución, los recursos económicos requeridos y disponibles, el análisis de requisitos de normatividades, la metodología para la ejecución del programa basada en la NOM-009-SEGOB-2015, la Ley General de Protección Civil y el Reglamento de la Ley General de Protección Civil, así como los recursos humanos capacitados. Al respecto es importante destacar que la Ley General de Protección Civil en su Art. 11 establece el requisito de que la persona autorizada para asesorar, capacitar, evaluar, realizar los estudios de vulnerabilidad y riesgos, elaborar los programas internos de protección civil y darles continuidad, debe contar con el registro de la autoridad competente 
de protección civil, ya que deberá emitir la carta de responsabilidad para la aprobación de los programas internos de protección civil. (LGPC 2020). Por este motivo se cuenta con la Participación del Centro Integral de Capacitación y Estudios de Seguridad e Higiene en el Trabajo, cuyo director es el Ing. Lino Alberto Gutiérrez Ramos.

\section{RESULTADOS}

De acuerdo con el análisis realizado se tiene un registro por parte de la Unidad Regional de Servicios Educativos Huasteca Norte (URSE) de 101 escuelas primarias en el municipio de Ciudad Valles y de 11 instituciones de educación primaria por parte del Sistema Educativo Estatal Regular (SEER), de las cuales 06 son oficiales y 05 particulares; en ninguno de los sistemas tienen conocimiento que es la protección civil, ni tienen desarrolladas actividades encaminadas a esos fines, tienen programas de convivencia escolar y suponen que podría ser "algo parecido" La URSE sostiene la referencia del área de recursos materiales quien se encarga de la infraestructura física, pero no la certeza de que se realizaran acciones de protección civil. Se investigó en la Coordinación Municipal de Protección Civil en Ciudad Valles cuantos registros de centros educativos de educación primaria tienen implementado un programa interno de protección civil y se encontró que solo 02 instituciones privadas cumplen con ese requisito. El colegio Mariano Arista y el Instituto Motolinía.

La Institución en estudio cuenta con una población estudiantil de 360 alumnos de los cuales 191 son niños y 169 niñas y 24 trabajadores que conforman el personal docente y administrativo; además de la participación de padres de familia y tutores que forman parte de la cotidianidad de las actividades escolares. En esta institución si conocen la guía para elaborar el programa interno de protección civil escolar del INIFED 2018, pero no se ha puesto en operación debido a los limitados recursos económicos, ya que al buscar asesoramiento profesional encontraron que tiene un costo.

Se realiza el diagnostico de vulnerabilidad y Evaluación de Riesgos encontrando riesgos relacionados con las condiciones de los techos, pisos, paredes, rampas y espacios libres. Los cuales presentan afectaciones que pueden ocasionar accidentes, provocando golpes, caídas, luxaciones. Riesgos eléctricos donde existen instalaciones incompletas e inseguras que pueden provocar cortos eléctricos o generar incendios o quemaduras. En relación con los riesgos provocados por amenazas naturales, se identifican el riesgo de inundación debido a las condiciones del suelo que presenta hundimientos y desniveles mal planeados. Riesgos por vientos fuertes que pueden provocar la caída de árboles, riesgos de almacenamiento de materiales de desecho no clasificados. Riesgos externos debido a las instalaciones eléctricas mal ubicadas. Referente al Mantenimiento preventivo y correctivo se atendieron gran parte 


\section{TECTZAPIC \\ Revista Académico-Científica}

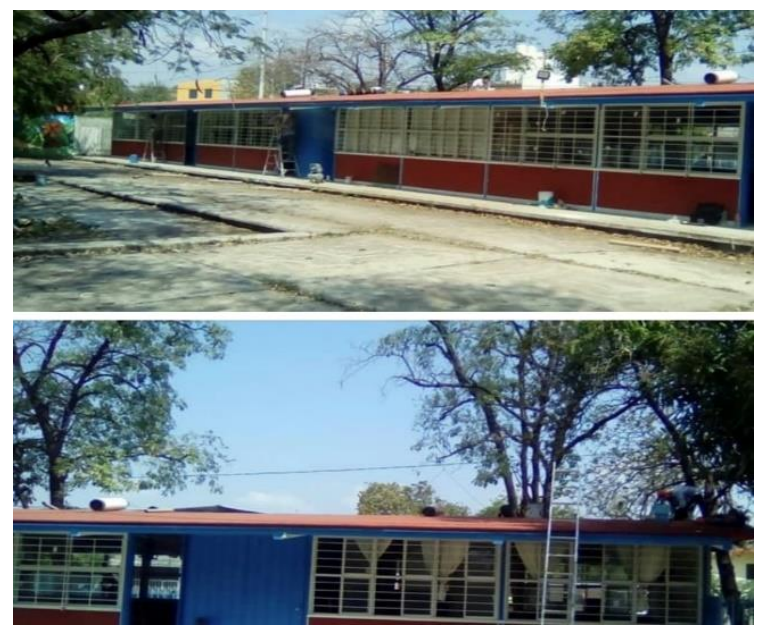

Figura 1. Mantenimiento de techos y pintura de Áreas
Revista de divulgación científica y tecnológica. ISSN: 2444-4944 Tecnológico Nacional de México Campus Ciudad Valles

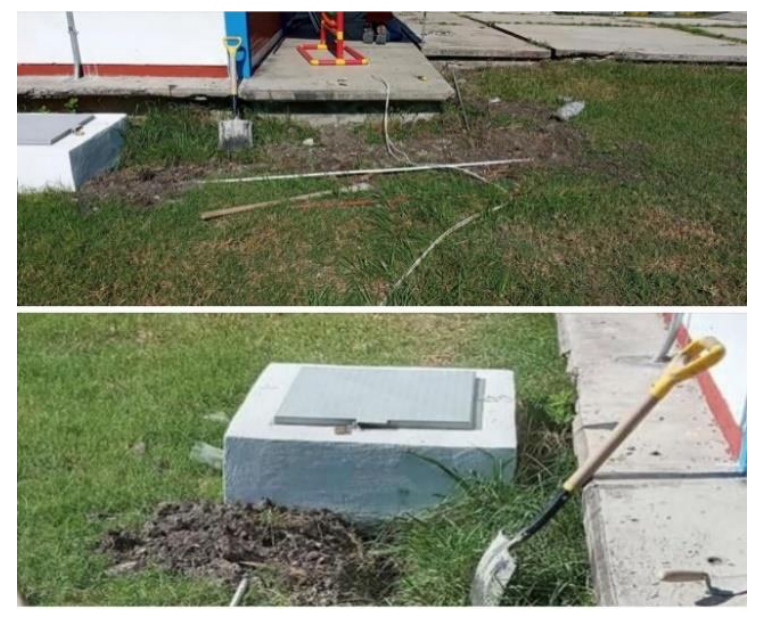

Figura 2. Mantenimiento a las Instalaciones

de las observaciones realizadas a la infraestructura física. Se cuenta con un solo extinguidor por lo que será necesario la adquisición de dos unidades más. El botiquín se requerirá actualizarlo y en cuanto al sistema de alertamiento habrá que adquirirlo. Se ha conformado la Unidad Interna con las brigadas correspondientes y se iniciaron los trabajos de capacitación para culminar con la ejecución de simulacros y los planes de contingencia. En cuanto a los planes de emergencia para acciones de Covid19 se establecieron los procedimientos para el monitoreo y detección. Por último, se calculó el presupuesto el cuál ronda en los 60,000 pesos sin considerar las erogaciones por mantenimiento, de los cuáles 42,000 pesos corresponden a la asesoría para la implementación del programa, la evaluación estructural y la capacitación mismos que serán patrocinados por el Centro Integral de Capacitación y Estudios de Seguridad e Higiene en el Trabajo, como una contribución altruista y se cuenta con la participación del Tecnológico Nacional de México campus Ciudad Valles.

\section{CONCLUSIONES}

Cada vez son más frecuentes y prominentes los eventos naturales y antropogénicos que generan grandes afectaciones a la población. En el ámbito escolar se considera que existe cierto nivel de seguridad ya que la construcción de los inmuebles se realiza en apego a la ley general de infraestructura física educativa, no obstante, las construcciones se deterioran, envejecen con el uso y el tiempo y requieren de mantenimientos que en muchas ocasiones no se realizan con la eficiencia que demandan por la falta y limitación de recursos económicos. Con esta investigación se pudo constatar lo alarmante que resulta que un tema de prioridad nacional como lo es la protección civil sea desconocido en los niveles de educación básica donde se encuentra la población estudiantil más vulnerable, por lo menos en la zona huasteca del estado de San Luis Potosí, destaca el hecho de que solo el 1.8\% de las Instituciones registradas tiene elaborado un plan de protección civil. Esta situación representa un desafío para las autoridades en los tres órdenes de gobierno y para quienes tienen la gobernanza de la educación básica en sus manos; generar e impulsar una cultura preventiva en Protección Civil gestionando la asesoría así como los recursos necesarios y difundiendo las acciones encaminadas a lograr espacios seguros, bien planeados, con planes de contingencias que respondan a las emergencias como la que estamos viviendo con la crisis sanitaria del covid 
19 y otras que se presentan de manera recurrente y con impactos muy severos en periodos muy cortos de tiempo.

\section{BIBLIOGRAFÍA}

Comité Internacional de la Cruz Roja CICR (1977) Protocolo I adicional a los Convenios de Ginebra de 1949 relativo a la protección de las víctimas de los conflictos armados internacionales, Ginebra Suiza

Comisión Nacional Forestal (2010) Incendios forestales Guía práctica para comunicadores (Tercera edición), Zapopan, Jal.

Instituto Nacional de la Infraestructura Física Educativa, Secretaria de Educación Pública, Subsecretaria de educación básica (2018); Guía para elaborar o actualizar el Programa Interno de Protección Civil (tercera edición) México D.F.

CENAPRED (2016) Que es el \#SINAPROC y cómo se consolido en nuestro país. Obtenida el 23 de septiembre del 2021 de https://www.gob.mx/cenapred/articulos/que-es-elsinaproc-y-como-se-consolido-en-nuetro-pais-enterate.

SSN, catálogo de sismos (2021): Universidad Nacional Autónoma de México, Instituto de Geofísica, Servicio Sismológico Nacional, México. Extraído el 22 de septiembre del 2021 desde http://www2.ssn.unam.mx:8080/catalogo/

Ley General de Protección Civil (2021) Última reforma de la Nueva Ley publicada en el Diario Oficial de la Federación el 6 de junio de 2012. Cámara de Diputados del $H$. Congreso de la Unión. México. Obtenido el 23 de septiembre del 2021 de http://www.diputados.gob.mx/LeyesBiblio/pdf/LGPC_200521.pdf

Cienciamx (2018), ocurren sismos en San Luis Potosí. Obtenido el 23 de septiembre del 2021 de http://www.cienciamx.com/index.php/ciencia/la-tierra/24337-ocurren-sismos-sanluis-potosi

UNICEF (2006), Convención sobre los derechos del niño 20 de noviembre de 1989 obtenido $\begin{array}{llllll}\text { el } & 23 & \text { de } & \text { septiembre } & \text { del } & 2021\end{array}$ https://www.un.org/es/events/childrenday/pdf/derechos.pdf

CNDH (2021), Incendio en la Guardería ABC obtenido el 24 de septiembre del 2021 de https://www.cndh.org.mx/noticia/incendio-en-la-guarderia-abc

CENAPRED (2020), 34 Aniversario del Sistema Nacional de Protección Civil obtenido el 23 de sep. de 2021 de https://www.gob.mx/cenapred/articulos/34-aniversario-delsistema-nacional-de-proteccion-civil

NOM-009-SEGOB-2015, (05 de 07 del 2018), Medidas de previsión, prevención y mitigación de riesgos en centros de atención infantil en la modalidad pública, privada y mixta. Diario Oficial de la Federación. México. Recuperado el 23 de sep. del 2021 de https://www.dof.gob.mx/nota_detalle.php?codigo=5530208\&fecha=05/07/2018 